\title{
Residual stresses in biaxially fatigued austenitic stainless steel sample of cruciform geometry
}

\author{
Yu V Taran ${ }^{1, *}$, A M Balagurov ${ }^{1}$, J Schreiber ${ }^{2}$, A Evans $^{3}$ and A M Venter ${ }^{4}$ \\ ${ }^{1}$ Frank Laboratory of Neutron Physics, Joint Institute for Nuclear Research, Dubna, \\ Russian Federation \\ ${ }^{2}$ Fraunhofer Institute for Nondestructive Testing (Dresden branch), Dresden, Germany \\ ${ }^{3}$ SINQ, Paul Scherrer Institute, Villigen, Switzerland \\ ${ }^{4}$ Research and Development Division, NECSA Limited, Pretoria, South Africa \\ *E-mail: taran@nf.jinr.ru
}

\begin{abstract}
A specifically designed cruciform-shaped austenitic stainless steel AISI 321 sample was subjected to ex-situ biaxial tension-compression cycling to establish ferromagnetic martensitic phase conversion under the action of plastic deformation. The time-of-flight neutron diffraction technique was employed for in-plane residual stress determination in this sample for both the austenitic and martensitic phases. The 2D data enabled determination of macro-, micro-, hydro- and deviatoric contributions to the total phase stresses.
\end{abstract}

\section{Introduction}

Investigation of fatigue degradation and martensitic transformation of austenitic stainless steel AISI 321 (Cr18Ni10Ti) is of great practical relevance due to its wide application amongst others in the nuclear and chemical industries. The experimental simulation of these processes is usually performed by uniaxial mechanical tension-compression cyclic loading. However, machines and structures are generally subjected to more complex multiaxial and possibly nonproportional stresses rather than simple uniaxial stresses. The failure and fracture problem under multiaxial stresses is one of the most important topics to ensure the reliability of industrial components.

To investigate the effects of in-plane biaxial loading on stress-induced martensite phase transformation, the cruciform geometry samples of two kinds named Krest and Kreuz were specifically designed for nondestructive investigation using neutron diffraction techniques. The design comprises a central membrane $15 \mathrm{~mm}$ in diameter and $2 \mathrm{~mm}$ thick surrounded by thicker material 5 $\mathrm{mm}$ in thickness extending to the cruciform edges. Specific care was taken to minimize possible sharp surfaces at the membrane-surrounding material interface to eliminate stress concentrators. Samples of this geometry were subjected to ex-situ biaxial out-of-phase tension-compression cycling under load control at different amplitudes using an Instron $100 \mathrm{kN}$ biaxial planar cruciform system. During plastic deformation a new ferromagnetic martensite phase is forming in the parent austenite matrix [1].

Sample Krest-2, cycled at $0.5 \mathrm{~Hz}$ frequency and $17 \mathrm{kN}$ load amplitude for 407 cycles rendering about $40 \%$ martensite volume fraction conversion, has been investigated earlier using time-of-flight (TOF) neutron diffraction techniques at the IBR-2 pulsed nuclear reactor at JINR [2, 3] along two perpendicular in-plane directions across the diameter of the membrane. A comprehensive 2D mapping could not be completed due to the IBR-2 facility being shut down for refurbishment. A 2D mapping of 
the membrane section was pursued following the (220) austenite and (211) martensite diffraction reflections using a monochromatic neutron beam at the ILL high flux reactor, the SALSA instrument. The residual stress analysis from the experimental data was inconclusive due to excessive crystallographic texture in the intensity of the martensite reflection [4]. Thus, the investigation of the Krest-2 was not finished. As distinct from the study of the Krest-2 the investigation of the Kreuz-1 was carried out to its conclusion in the present work.

To overcome the problems associated with the crystallographic texture, it became essential that a number of diffraction peaks representative of each phase be investigated simultaneously to assist in the identification of those Bragg reflections for which the intensities were not influenced to adversely. This paper subsequently reports the results from a 2D diffraction analysis of the in-plane residual stresses in the membrane section of the Kreuz-1 sample, using TOF neutron diffraction at the SINQ spallation source (Paul Scherer Institute, Switzerland), the POLDI instrument. POLDI is a correlation type TOF diffractometer [5].

\section{Experiment}

The Kreuz-1 sample (Fig. 1) has been subjected to biaxial cycling at load amplitude of $15 \mathrm{kN}$ and frequency of $0.5 \mathrm{~Hz}$ until rupture of the membrane. This rupture occurred during the tension cycle 1904 within two adjoining quarters of $A 2-0-B 1$ and $A 2-0-B 2$ at the interface of the membrane and the peripheral 5-mm-thick surrounding material as indicated in Fig. 1.

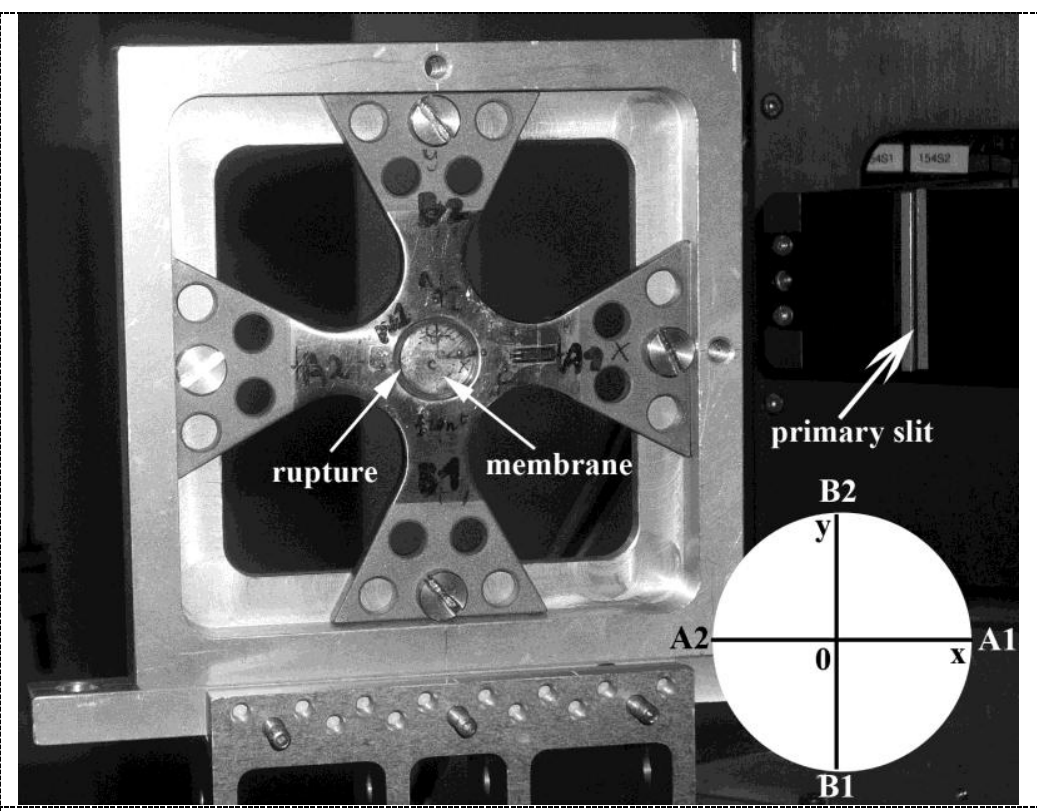

Figure 1. The Kreuz-1 sample mounted on the POLDI instrument for the in-plane $x$-component measurements. The right bottom insert is shown the membrane image with the sample coordinate system $(x, y)$ and the loading directions $A 1-A 2$ and $B 1-B 2$. The neutron scattering vector was along the loading direction $A 1-A 2$. The vertical slit is visible at the right top with the horizontal slit directly behind it. The $y$-component measurements were done by rotating the sample $90^{\circ}$ clockwise to have this direction along the scattering vector. 
The experimental set-up of the Kreuz-1 cruciform sample on the POLDI instrument is shown in Fig. 1. A neutron gauge volume fully illuminating the $2 \mathrm{~mm}$ membrane thickness, but limited to a size of 1.5 $\mathrm{mm}$ vertically and horizontally, was formed by using slits $1.5 \mathrm{~mm}$ in size for the primary neutron beam and a $4 \mathrm{~mm}$ radial collimator in the secondary neutron beam. Diffraction data were recorded by a one-dimensional ${ }^{3} \mathrm{He}$ position-sensitive detector covering an angular range of nearly $30^{\circ}$ at a central scattering angle $2 \theta=90^{\circ}$. The $2 \mathrm{D}$ in-plane diffraction mapping of the membrane and the surrounding material was performed using a discrete $1 \times 1 \mathrm{~mm}^{2}$ rectangular measurement mesh. The sample coordinate system $(x, y)$ corresponds to the loading directions $A 1-A 2$ and $B 1-B 2$, respectively (both systems are shown in the insert to Fig. 1).

Strain scanning was performed on the two quarters of $A 1-0-B 2$ (right) and $A 2-0-B 2$ (left) assuming mirror symmetry relative to the $A 1-0-A 2$ axis. Diffraction measurements were carried out for two perpendicular orientations of the sample with the neutron scattering vector being directed along either the $x$ - or $y$-axis ( $x$ - or $y$-component hereinafter). Neutron diffraction patterns covering the $d$-spacing range 0.7-2.2 $\AA$ were measured at all sampling points. Selective measurements at points surrounding the membrane section were used as reference for the strain assessment in the austenite phase since no martensite formation was present here. Data processing was done on the (111) austenite and (110) martensite diffraction reflections using a Gaussian single-peak fit approach (POLDI in-house program "Poldiausfit").

\section{Total austenite residual stress tensor}

To calculate residual strains from the experimentally measured $d$-values of the (111) austenite and (110) martensite peaks respective stress-free reference $d$-spacing is needed. Unfortunately, owing to the complex nature of the austenite matrix phase transformation during fatigue cycling, it is impossible to obtain stress-free data for martensite inclusions. For the austenite phase, as a rough but plausible approach, the unstrained reference parameter $d_{0}(111)=2.072418 \AA$ was measured on the outer part of the sample surrounding the membrane where no plastic yielding and martensite phase formation had occurred. Using Hooke's law for a biaxial geometry, the austenite stress tensor in each measured point was calculated using Young's modulus $E=170 \mathrm{GPa}$ and Poisson ratio $v=0.3$. In Fig. 2 both $x$ - and $y$ components of the austenite total residual stresses are illustrated (two upper curves) as a function of radius $r$ along the $A 1-0-A 2$ diameter line $(y=0)$. The qualitative estimation of the stress relief occurring in the austenite phase due to the rupture of the membrane was obtained by averaging the $x$ - and $y$ components of the total stresses in both investigated quarters. The calculated values were within 40 $\mathrm{MPa}$, implying that the stress relief in the austenite phase is marginal. 


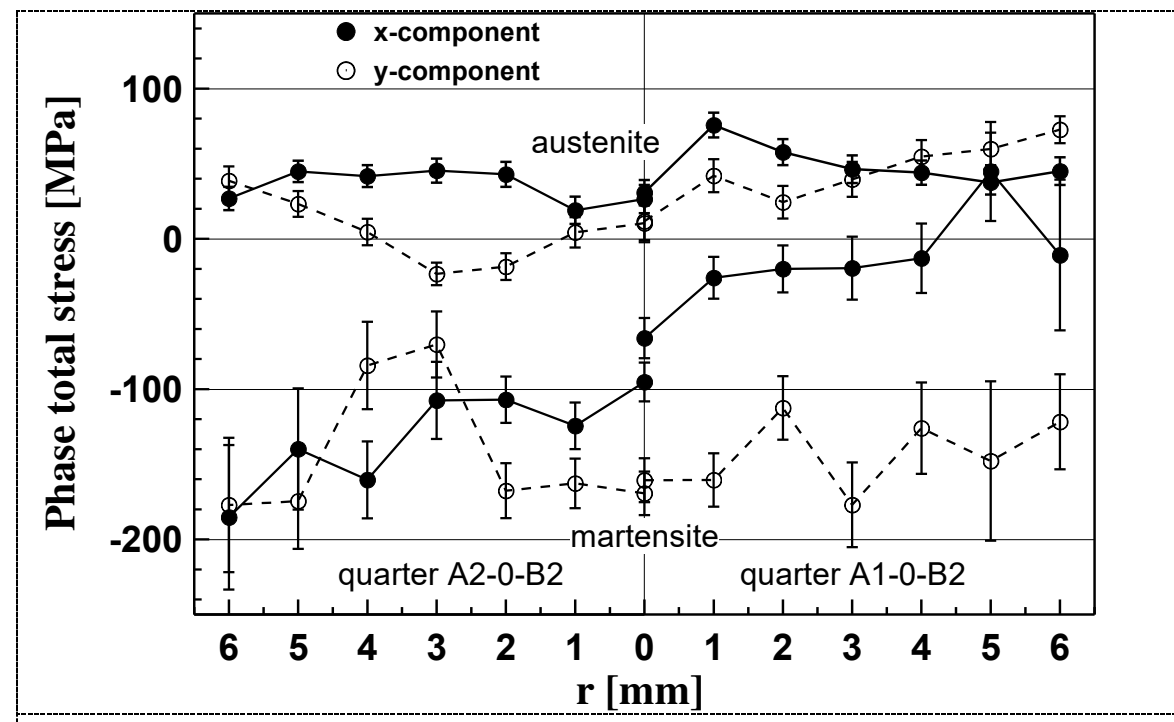

Figure 2. Radial distributions of $x$ - and $y$-components of the phase total residual stresses in austenite (two upper curves) and martensite (two lower curves) as a function of radius $r$ along the A1-0-A2 diameter line with $y=0$.

\section{Solution of $\boldsymbol{d}_{0}$-problem for martensite phase}

For the determination of the martensite unstrained parameter, $d_{0}(110)$, three general conditions can be applied (e.g., see [6,7]), namely, separation of the total stress, ${ }^{t} \sigma$, into macrostress, ${ }^{M} \sigma$ (the same in each phase) and phase microstress, ${ }^{\mu} \sigma$, a phase mixture rule and a stress equilibrium approach, which successively are:

$$
\begin{gathered}
{ }^{t} \sigma_{j}^{p}={ }^{M} \sigma_{j}+{ }^{\mu} \sigma_{j}^{p}, \\
{ }^{\mu} \sigma_{j}^{a} f^{a}+{ }^{\mu} \sigma_{j}^{m} f^{m}=0, \\
{ }^{M} \bar{\sigma}_{j}=\frac{1}{V} \int_{V}{ }^{M} \sigma_{j} d V=0,
\end{gathered}
$$

where subscript $j$ indicates a stress component at a sampled point, $f^{p}$ represents a volume fraction of $p$-phase, $p=a$ for austenite or $m$ for martensite, respectively. Using Eqs. (1), (2), the macrostress can be expressed as:

$$
{ }^{M} \sigma_{j}={ }^{t} \sigma_{j}^{a} f^{a}+{ }^{t} \sigma_{j}^{m} f^{m} .
$$

As the measurements were made in a discrete mesh, the integration over a free body volume can be replaced by the summation

$$
\sum_{k}\left[{ }^{t} \sigma_{j}^{a}\left(\vec{r}_{k}\right) f^{a}\left(\vec{r}_{k}\right)+{ }^{t} \sigma_{j}^{m}\left(\vec{r}_{k}\right) f^{m}\left(\vec{r}_{k}\right)\right]=0,
$$

where the radius-vector, $\vec{r}_{k}$, corresponds to the $k$-th mesh cell.

If the phase volume fractions are known, the martensite unstrained parameter, $d_{0}(110)$, may be estimated from Eq. (5) using the planar presentation of Hooke's law for the total martensite stress ${ }^{t} \sigma_{j}^{m}\left(\vec{r}_{k}\right)$ expressed through the martensite lattice spacing $d(110)$. 


\section{Determination of martensite phase volume fraction}

Taking into account that $f^{a}+f^{m}=1$, the martensite volume fraction in each sampled point was calculated from the ratio of the austenite and martensite diffraction peak areas:

$$
A_{a} / A_{m}=\left(f^{a} / f^{m}\right)\left(F_{a} / \mathrm{F}_{\mathrm{m}}\right)^{2}\left(j_{a} / j_{m}\right)\left(Q_{a} / Q_{m}\right),
$$

where $p$-phase values $F_{p}$ and $j_{p}$ are the structure factor and reflection multiplicity. For the $Q$ factor it can be shown that in the case of TOF diffraction the relation $Q_{p} \sim\left(1 / d_{p}\right)^{2}$ is valid. The results of martensite fraction calculations for $x$ - and $y$-components are shown in Fig. 3.

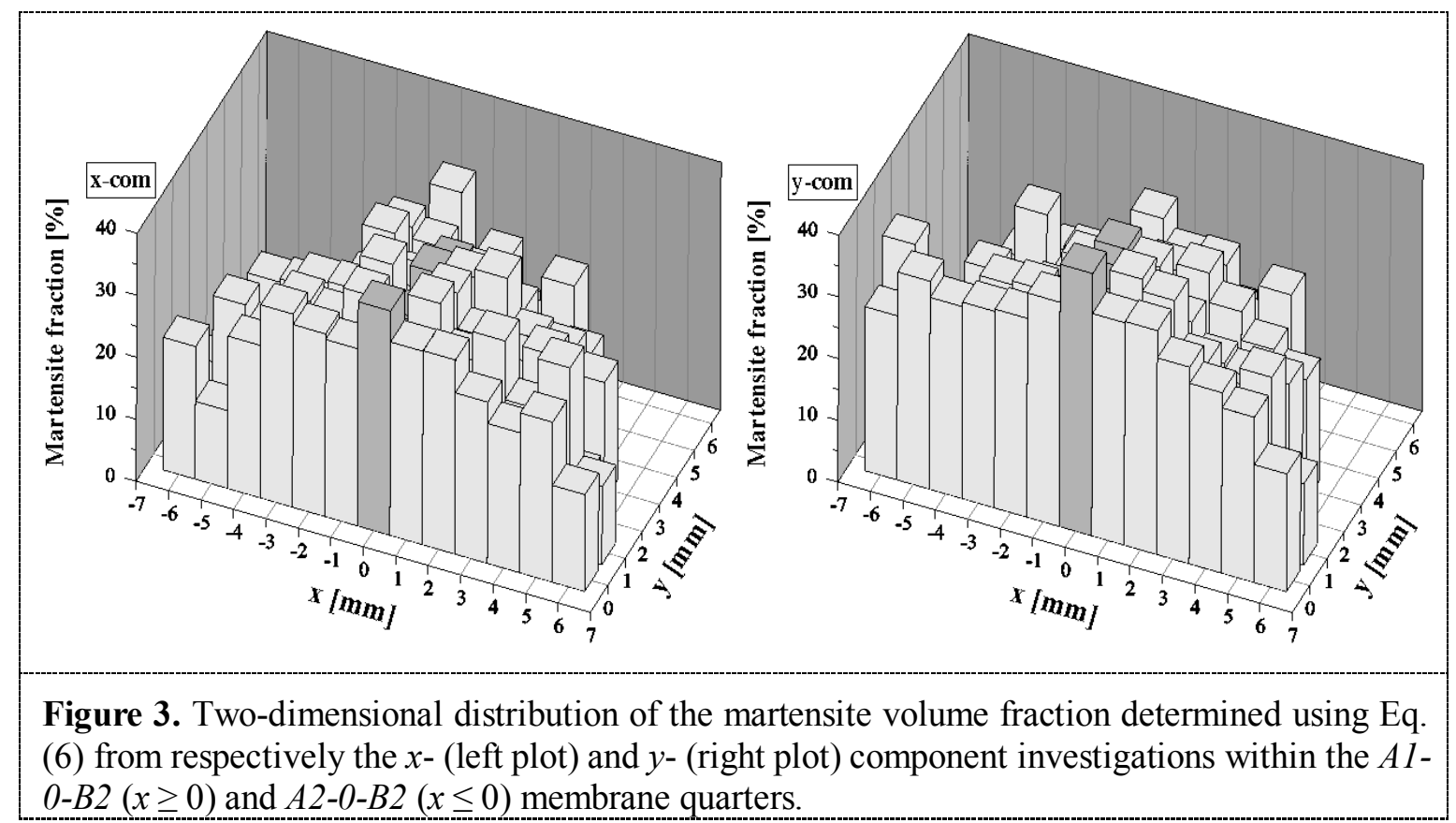

As a first-order observation, some asymmetry seems to exist between the left and right membrane quarters. However, the mean values of martensite fraction within both quarters summarized in Table 1 do not show any marked asymmetry. Despite the rupture of the membrane in the left quarter, asymmetry is not expected as the martensite transformation is irreversible. The $4 \%$ difference between the mean values of martensite fraction for the $x$ - and $y$-components can be ascribed to the presence of weak crystallographic texture in the (110) martensite intensity.

Table 1. Experimental data for martensite phase.

\begin{tabular}{|c|c|c|c|c|c|c|}
\hline \multirow{2}{*}{ quarter } & \multicolumn{3}{|c|}{$x$-component } & \multicolumn{3}{c|}{$y$-component } \\
\cline { 2 - 7 } & fraction $[\%]$ & $d_{0}(110)[\AA]$ & ${ }^{t} \sigma_{x}^{m}[\mathrm{MPa}]$ & fraction $[\%]$ & $d_{0}(110)[\AA]$ & ${ }^{t} \sigma_{y}^{m}[\mathrm{MPa}]$ \\
\hline right & 23.8 & 2.030077 & $-58.2(8.4)$ & 27.5 & 2.029965 & $-120.9(5.8)$ \\
\hline left & 23.7 & 2.029929 & $-112.9(9.4)$ & 26.7 & 2.029432 & $-114.7(7.6)$ \\
\hline
\end{tabular}

\section{Total martensite residual stress tensor}

Assuming no elastic mismatch between austenite and martensite phases and resolving Eq. (5) relative to $d_{0}(110)$, four $d_{0}$-values were respectively determined from the $\mathrm{x}$ - and $\mathrm{y}$-component measurements in both quarters (Table 1). The mean value, $2.029851 \AA$, was subsequently used as the 
martensite unstrained reference parameter $d_{0}(110)$ for the strain calculations. The martensite stress tensor in each measured point was calculated taking the elastic constants as used for the austenite phase. In the case, we have neglected elastic anisotropy in each phase. In Fig. 2 (two lower curves) both $x$ - and $y$-components of the martensite total residual stresses are illustrated as a function of radius $\mathrm{r}$ along the $A 1-0-A 2$ diagonal line for $y=0$. The averaged martensite total residual stresses within each quarter are shown in Table $1\left({ }^{t} \sigma_{x}^{m}\right.$ and $\left.{ }^{t} \sigma_{y}^{m}\right)$. A large difference exists in the values between the left and right membrane quarters for the $x$-component, while the $y$-component values are similar. This is indicative of potential stress relief in the martensite phase due to the rupture in the membrane.

\section{Separation of macrostress from total phase residual stresses}

By using Eq. (4), the residual macrostresses can be separated as ${ }^{M} \sigma_{x}$ and ${ }^{M} \sigma_{y}$ in a two-phase material. The 2D results of the $x$ - and $y$-component macrostresses within the top half of the membrane are shown in Fig. 4 from diffraction measurements parallel to the A1-0-A2 direction and for $y$ coordinate between 0 and $6 \mathrm{~mm}$. The $x$-component (Fig. 4, left plot) demonstrates noticeable asymmetry between the right and left quarters. Its value, as such, in the quarter $A 1-0-B 2$ varies from 60 to $-40 \mathrm{MPa}$, while its dispersion in the quarter $A 2-0-B 2$ is appreciably smaller varying between 35 to $-20 \mathrm{MPa}$. Again this effect may be attributed to the stress relief of the $x$-macrostress component due to the membrane rupture. The $y$-component macrostress does not show obvious asymmetry between the quarters.

The residual macrostress calculations are based on the macrostress equilibrium condition (Eq. (3)), i.e., the mean values of the $x$ - and $y$-components of macrostresses in both membrane quarters must equal zero. The results from the averaging macrostress procedure are presented in Table 2 . The fact that the averaged macrostress components are not exactly equal to zero, but within $17 \mathrm{MPa}$, reflects the approximate nature of the assumptions used for the determination of phase strain-free parameters being satisfactory. This particularly concerns determination of the $d_{0}$-parameter for the martensite phase, potentially influenced by texture contributions on the martensite volume fraction parameter used in the calculations.
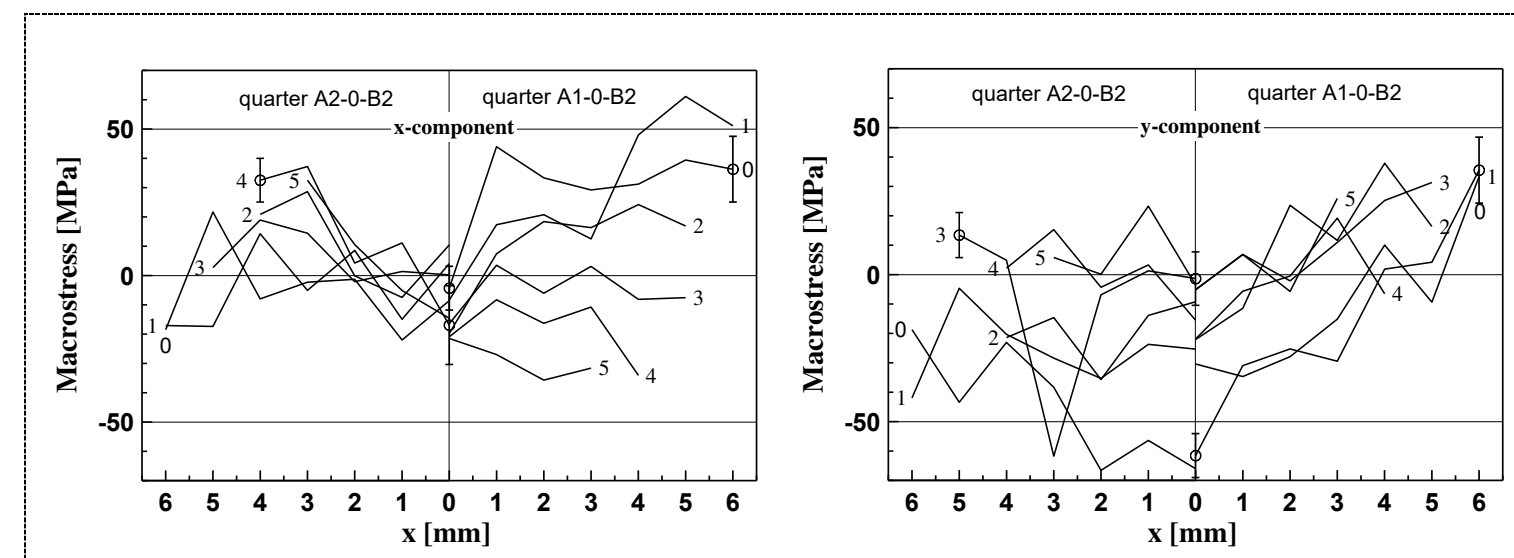

Figure 4. Dependences of the $x$ - and $y$-component macrostresses (left and right plots, respectively) on $x$-coordinate in the top half section of the membrane at different $y$-coordinates shown as a number of a curve. A typical error is shown for some curves. 
Table 2. Macro- and hydrostresses.

\begin{tabular}{|c|c|c|c|c|}
\hline \multirow{2}{*}{ quarter } & \multicolumn{2}{|c|}{ averaged macrostress [MPa] } & \multicolumn{2}{c|}{ averaged hydrostress [MPa] } \\
\cline { 2 - 5 } & $x$-comp. & $y$-comp. & austenite & martensite \\
\hline right & $6.8(4.5)$ & $-1.4(3.9)$ & $22.1(2.4)$ & $-59.7(3.4)$ \\
\hline left & $2.5(2.8)$ & $-17(4)$ & $18.7(1.4)$ & $-75.8(4.8)$ \\
\hline
\end{tabular}

\section{Separation of microstress from total phase residual stresses}

In addition to macrostresses, Eqs. (1), (2) allow the calculation of the planar components of the phase microstresses:

$$
\begin{aligned}
& { }^{\mu} \sigma_{j}^{a}=f^{m}\left({ }^{t} \sigma_{j}^{a}-{ }^{t} \sigma_{j}^{m}\right), \\
& { }^{\mu} \sigma_{j}^{m}=-f^{a}\left({ }^{t} \sigma_{j}^{a}-{ }^{t} \sigma_{j}^{m}\right) .
\end{aligned}
$$

As an illustration, the results of the microstress $x$ - and $y$-component calculations for the austenite and martensite phases are shown in Fig. 5. This shows that the austenite phase is in a state of tension, whilst the martensite phase exhibits a balancing compressive stress with the larger value being in accordance with its smaller volume fraction. The asymmetry in the microstresses of the martensite phase between the left and right quarters of the membrane may be attributed to the crystallographic texture.

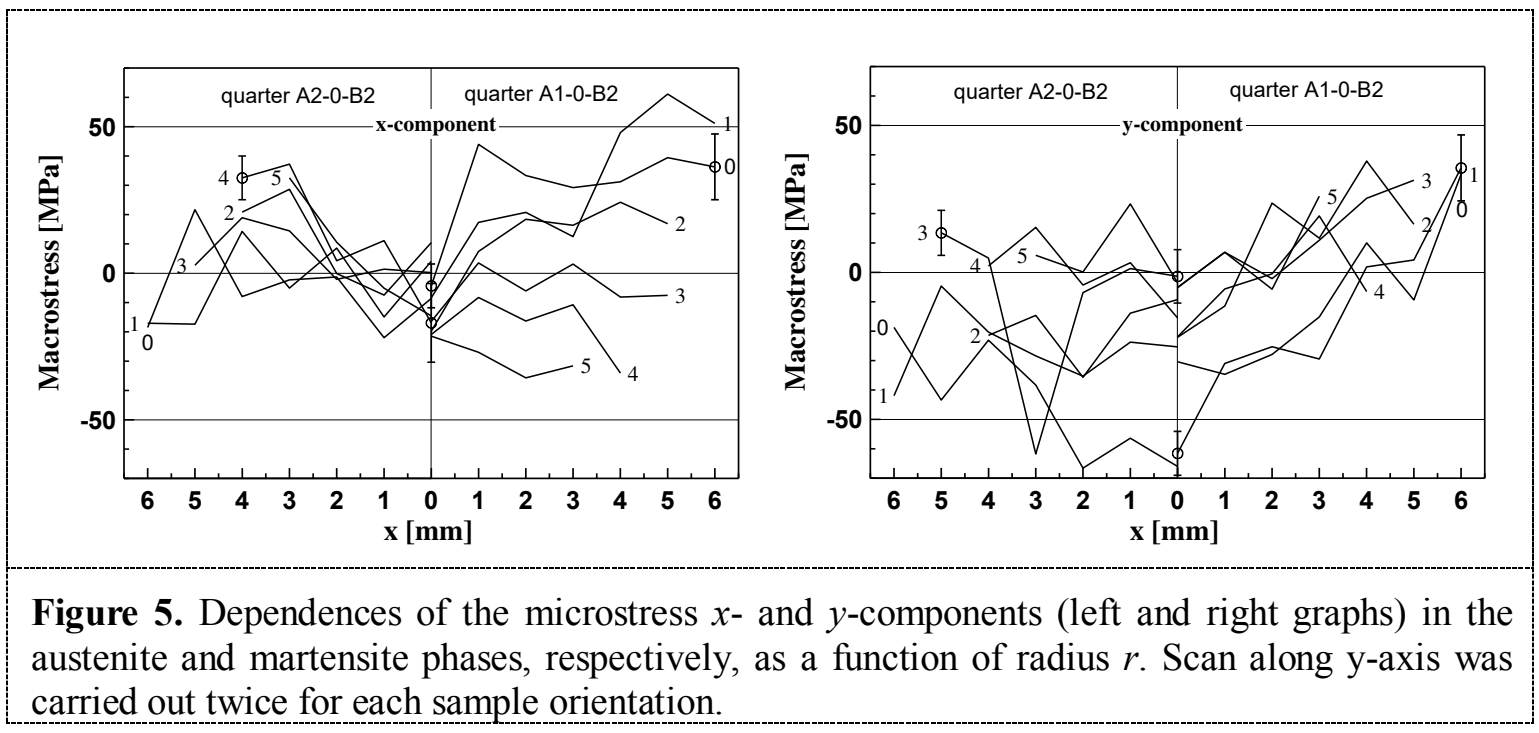

\section{Separation of hydrostatic stress from total phase residual stresses}

Since the plastic deformation does not occur under hydrostatic (volumetric) stress, the deviatoric stress tensor can adequately describe the residual stress state with respect to mechanical loading such as fatigue cycling. The total stress tensor of a $p$-phase may be separated into the hydrostatic $\tau_{H}^{p}$ and deviatoric ${ }^{t} \tau_{j}^{p}$ components:

$$
{ }^{t} \sigma_{j}^{p}={ }^{t} \tau_{H}^{p}+{ }^{t} \tau_{j}^{p},
$$

where, by definition, $\tau_{H}^{p}=(1 / 3) \cdot \operatorname{Tr}\left({ }^{t} \sigma_{i}^{p}\right)$ and $\operatorname{Tr}\left({ }^{t} \tau_{i}^{p}\right)=0$. The phase hydrostatic components $\tau_{H}^{p}$ calculated using the results of the planar total residual stresses of austenite and martensite phases are 
shown in Fig. 6. The results of the averaging hydrostress procedure presented in Table 2 point to practical absence of asymmetry between the right and left membrane quarters.

It is known that the martensite formation during plastic deformation of an austenite matrix is connected with volume dilatation. Since the specific volume of martensite is larger (about $2 \%$ ) than that of austenite, the martensite phase is generally expected to be in hydrostatic compression, whereas the austenite is in tension. Since the deviatoric stress tensor is a simple difference between the total and hydrostatic stress tensors, we do not present the result of its calculation.

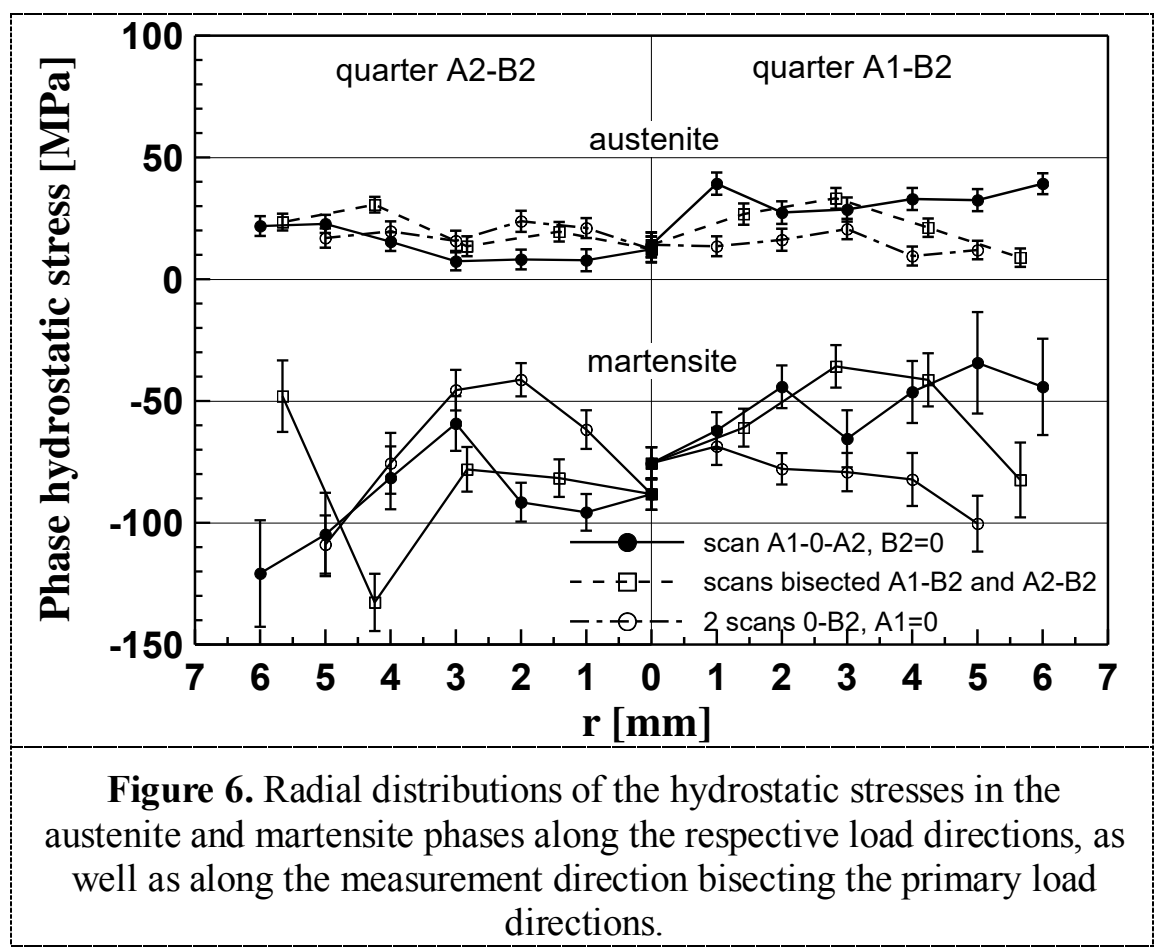

\section{Conclusion}

The main results of this work:

1) The biaxial fatigue cycling of austenitic stainless steel AISI 321 was performed in ex-situ on a sample of cruciform geometry with an Instron $100 \mathrm{kN}$ biaxial planar cruciform system. The martensite transformation of austenite steel matrix was observed during plastic deformation.

2) Neutron diffraction 2D-strain scanning and residual stress determination for both the austenite and martensite phases were carried out with the time-of-flight high resolution stress-diffractometer POLDI.

3) Separation of the phase total residual stresses into macro-, micro-, hydro- and deviatoric residual stresses was possible using a phase mixture rule and a stress equilibrium approach.

The authors are grateful to the SINQ facility for the allocation of beam time at the POLDI instrument, as well as to Prof. R.A.Winholtz from the University of Missouri (USA) for very useful consultations. The financial support from the JINR-SA Bilateral Scientific Cooperation Programme is gratefully acknowledged. 
The authors are grateful to the SINQ facility (PSI) for the allocation of beam time at the POLDI instrument. The financial support from the JINR-SA Bilateral Scientific Cooperation Programme is gratefully acknowledged.

\section{References}

[1] Taran Yu.V. et al. Investigation of In-Plane Biaxial Low Cycle Fatigued Austenitic Stainless Steel AISI 321. I. Mechanical Testing on the Planar Biaxial Load Machine. JINR Commun. E18-2007-110. Dubna, 2007. 24 p.

[2] Taran Yu.V. et al. Investigation of In-Plane Biaxial Low Cycle Fatigued Austenitic Stainless Steel AISI 321. II. Neutron Diffraction Stress Analysis at the IBR-2 Pulsed Nuclear Reactor. JINR Commun. E18-2007-111. Dubna, 2007. 18 p.

[3] Taran Yu.V. et al. Neutron Diffraction Analysis of Stresses in an In-Plane Biaxially-Fatigued Stainless Steel Sample of Cruciform Geometry. Materials Sci. Forum. 2008. V. 571 -572. P. 131-136.

[4] Taran Yu.V. et al. Neutron Diffraction Research of In-Plane Biaxial Fatigued Stainless Steel Sample of Cruciform Geometry. The ILL Experimental Report No. 1-01-29. 2008; http://club.ill.fr/cv/.

[5] Stuhr U. et al. Time-of-Flight Diffraction with Multiple Frame Overlap. Part II: The Strain Scanner POLDI at PSI. Nucl. Instr. Meth. A. 2005. V. 545. P. 330-338.

[6] Winholtz R.A, Cohen J.B. Load Sharing of the Phases in 1080 Steel during Low-Cycle Fatigue. Metallurg. Transactions A. 1992. V. 23. P. 341-354.

[7] Winholtz R.A. Separation of Microstresses and Macrostresses. Measurements of Residual and Applied Stress Using Neutron Diffraction. Ed. by M.T.Hutchings and A.D.Krawitz. Kluwer Academic Publishers, Dordrecht, 1992. P. 131. 